\title{
Multiplicative order convergence in $f$-algebras
}

\author{
Abdullah Aydın (1) \\ Department of Mathematics, Muş Alparslan University, Muş, Turkey
}

\begin{abstract}
A net $\left(x_{\alpha}\right)$ in an $f$-algebra $E$ is said to be multiplicative order convergent to $x \in E$ if $\left|x_{\alpha}-x\right| u \stackrel{\mathrm{o}}{\rightarrow} 0$ for all $u \in E_{+}$. In this paper, we introduce the notions mo-convergence, moCauchy, mo-complete, mo-continuous, and mo-KB-space. Moreover, we study the basic properties of these notions.
\end{abstract}

Mathematics Subject Classification (2010). 46A40, 46E30

Keywords. mo-convergence, $f$-algebra, mo-KB-space, vector lattice

\section{Introductory facts}

In spite of the nature of the classical theory of Riesz algebra and $f$-algebra, as far as we know, the concept of convergence in $f$-algebras related to multiplication has not been done before. However, there are some close studies under the name unbounded convergence in some kinds of vector lattices; see for example [2-6]. In the light of this information, we define a new concept of the convergence, which is called the mo-convergence, on $f$-algebras. Our aim is to introduce the concept of the mo-convergence by using the multiplication in $f$-algebras and examine the relationship between other types of convergence.

First of all, let us remember some notations and terminologies used in this paper. Let $E$ be a real vector space. Then $E$ is called ordered vector space if it has an order relation $\leq$ (i.e, $\leq$ is reflexive, antisymmetric, and transitive) that is compatible with the algebraic structure of $E$ that means $y \leq x$ implies $y+z \leq x+z$ for all $z \in E$ and $\lambda y \leq \lambda x$ for each $\lambda \geq 0$. An ordered vector $E$ is said to be vector lattice (or, Riesz space) if, for each pair of vectors $x, y \in E$, the supremum $x \vee y=\sup \{x, y\}$ and the infimum $x \wedge y=\inf \{x, y\}$ both exist in E. Moreover, $x^{+}:=x \vee 0, x^{-}:=(-x) \vee 0$, and $|x|:=x \vee(-x)$ are called the positive part, the negative part, and the absolute value of $x \in E$, respectively. Also, two vectors $x, y$ in a vector lattice are said to be disjoint whenever $|x| \wedge|y|=0$. A vector lattice $E$ is called order complete if $0 \leq x_{\alpha} \uparrow \leq x$ implies the existence of $\sup x_{\alpha}$ in $E$. A subset $A$ of a vector lattice is called solid whenever $|x| \leq|y|$ and $y \in A$ imply $x \in A$. A solid vector subspace is referred to as an order ideal. An order closed ideal is referred to as a band. A sublattice $Y$ of a vector lattice is majorizing $E$ if, for every $x \in E$, there exists $y \in Y$ with $x \leq y$. A partially ordered set $I$ is called directed if, for each $a_{1}, a_{2} \in I$, there is another $a \in I$ such that $a \geq a_{1}$ and $a \geq a_{2}$ (or, $a \leq a_{1}$ and $a \leq a_{2}$ ). A function from a directed set $I$ into a set $E$ is called a net in $E$. A net $\left(x_{\alpha}\right)_{\alpha \in A}$ in a vector lattice $X$ is called order convergent (or shortly, o-convergent) to $x \in X$, if there exists another net

Email address: aaydin.aabdullah@gmail.com

Received: 11.01.2019; Accepted: 19.06.2019 
$\left(y_{\beta}\right)_{\beta \in B}$ satisfying $y_{\beta} \downarrow 0$, and for any $\beta \in B$ there exists $\alpha_{\beta} \in A$ such that $\left|x_{\alpha}-x\right| \leq y_{\beta}$ for all $\alpha \geq \alpha_{\beta}$. In this case, we write $x_{\alpha} \stackrel{o}{\rightarrow} x$; for more details see for example $[1,7,8]$.

A vector lattice $E$ under an associative multiplication is said to be a Riesz algebra whenever the multiplication makes $E$ an algebra (with the usual properties), and in addition, it satisfies the following property: $x, y \in E_{+}$implies $x y \in E_{+}$. A Riesz algebra $E$ is called commutative if $x y=y x$ for all $x, y \in E$. A Riesz algebra $E$ is called $f$-algebra if $E$ has additionally property that $x \wedge y=0$ implies $(x z) \wedge y=(z x) \wedge y=0$ for all $z \in E_{+}$; see for example [1]. A vector lattice $E$ is called Archimedean whenever $\frac{1}{n} x \downarrow 0$ holds in $E$ for each $x \in E_{+}$. Every Archimedean $f$-algebra is commutative; see Theorem 140.10 [8]. Assume $E$ is an Archimedean $f$-algebra with a multiplicative unit vector $e$. Then, by applying Theorem 142.1(v) [8], in view of $e=e e=e^{2} \geq 0$, it can be seen that $e$ is a positive vector. In this article, unless otherwise stated, all f-algebras are semiprime, and all vector lattices are assumed to be real and Archimedean, and so $f$-algebras are commutative.

Recall that a net $\left(x_{\alpha}\right)$ in a vector lattice $E$ is unbounded order convergent (or shortly, uoconvergent) to $x \in E$ if $\left|x_{\alpha}-x\right| \wedge u \stackrel{o}{\rightarrow} 0$ for every $u \in E_{+}$. In this case, we write $x_{\alpha} \stackrel{u o}{\longrightarrow} x$; see for example [6] and [2-4]. Motivated from this definition, we give the following notion.

Definition 1.1. Let $E$ be an $f$-algebra. A net $\left(x_{\alpha}\right)$ in $E$ is said to be multiplicative order convergent to $x \in E$ (shortly, $\left(x_{\alpha}\right)$ mo-converges to $x$ ) if $\left|x_{\alpha}-x\right| u \stackrel{\circ}{\rightarrow} 0$ for all $u \in E_{+}$. Abbreviated as $x_{\alpha} \stackrel{\mathrm{mo}}{\longrightarrow} x$.

It is clear that $x_{\alpha} \stackrel{\mathrm{mo}}{\longrightarrow} x$ in an $f$-algebra $E$ implies $x_{\alpha} y \stackrel{\mathrm{mo}}{\longrightarrow} x y$ for all $y \in E$ because of $|x y|=|x||y|$ for all $x, y \in E$. Also, in general, the mo-convergence and uo-convergence are not the same. To see that we consider the following example.

Example 1.2. Let $E$ be a vector lattice and consider $\operatorname{Orth}(E):=\left\{T \in L_{b}(E): x \perp\right.$ $y$ implies $T x \perp y\}$ the set of orthomorphisms on $E$. The space $\operatorname{Orth}(E)$ is not only vector lattice but also an $f$-algebra. The mo-convergence and the uo-convergence are different in $\operatorname{Orth}(E)$.

We shall keep in mind the following useful lemma, obtained from the property of $x y \in$ $E_{+}$for every $x, y \in E_{+}$.

Lemma 1.3. If $y \leq x$ is provided in an f-algebra $E$ then $u y \leq u x$ for all $u \in E_{+}$.

Recall that multiplication by a positive element in $f$-algebras is a vector lattice homomorphism, i.e., $u(x \wedge y)=(u x) \wedge(u y)$ and $u(x \vee y)=(u x) \vee(u y)$ for every positive element $u$; see for example Theorem 142.1(i) [8]. We will denote an l-algebra $E$ as infinite distributive l-algebra whenever the following condition holds: if $\inf (A)$ exists for any subset $A$ of $E_{+}$then the infimum of the subset $u A$ exists and $\inf (u A)=u \inf (A)$ for each positive vector $u \in E_{+}$. For a net $\left(x_{\alpha}\right) \downarrow 0$ in an infinite distributive $l$-algebra, the net $\left(u x_{\alpha}\right)$ is also decreasing to zero for all positive vector $u$. Fortunately, every $f$-algebra has the infinite distributive property.

Remark 1.4. The order convergence implies the mo-convergence in $f$-algebras. The converse holds true in $f$-algebras with multiplication unit. Indeed, assume a net $\left(x_{\alpha}\right)_{\alpha \in A}$ order converges to $x$ in an $f$-algebra $E$. Then there exists another net $\left(y_{\beta}\right)_{\beta \in B}$ satisfying $y_{\beta} \downarrow 0$, and, for any $\beta \in B$, there exists $\alpha_{\beta} \in A$ such that $\left|x_{\alpha}-x\right| \leq y_{\beta}$. Hence, we have $\left|x_{\alpha}-x\right| u \leq y_{\beta} u$ for all $\alpha \geq \alpha_{\beta}$ and for each $u \in E_{+}$. Since $y_{\beta} \downarrow$, we have $u y_{\beta} \downarrow$ for each $u \in E_{+}$by Lemma 1.3, and $\inf \left(u y_{\beta}\right)=u \inf \left(y_{\beta}\right)=0$ because of $\inf \left(y_{\beta}\right)=0$. Therefore, $\left|x_{\alpha}-x\right| u \stackrel{\mathrm{o}}{\rightarrow} 0$ for each $u \in E_{+}$. That means $x_{\alpha} \stackrel{\text { mo }}{\longrightarrow} x$.

For the converse, assume $E$ is an $f$-algebra with multiplication unit $e$ and $x_{\alpha} \stackrel{\text { mo }}{\longrightarrow} x$ in $E$. That is, $\left|x_{\alpha}-x\right| u \stackrel{\circ}{\rightarrow} 0$ for all $u \in E_{+}$. Since $e \in E_{+}$, in particular, choose $u=e$, and so we have $\left|x_{\alpha}-x\right|=\left|x_{\alpha}-x\right| e \stackrel{\mathrm{o}}{\rightarrow} 0$, or $x_{\alpha} \stackrel{\circ}{\rightarrow} x$ in $E$.

By considering Example 141.5 [8], we give the following example. 
Example 1.5. Let $[a, b]$ be a closed interval in $\mathbb{R}$ and let $E$ be vector lattice of all reel continuous functions on $[a, b]$ such that the graph of functions consists of a finite number of line segments. In view of Theorem 141.1 [8], every positive orthomorphism $\pi$ in $E$ is trivial orthomorphism, i.e., there is a reel number $\lambda$ such that $\pi(f)=\lambda f$ for all $f \in E$. Therefore, a net of positive orthomorphism $\left(\pi_{\alpha}\right)$ is order convergent to $\pi$ if and only if it is mo-convergent to $\pi$ whenever the multiplication is the natural multiplicative, i.e., $\pi_{1} \pi_{2}(f)=\pi_{1}\left(\pi_{2} f\right)$ for all $\pi_{1}, \pi_{2} \in \operatorname{Orth}(E)$ and all $f \in E$. Indeed, $\operatorname{Orth}(E)$ is Archimedean $f$-algebra with the identity operator as a unit element; see Theorem 140.4 [8]. So, by applying Remark 1.4, the mo-convergence implies the order convergence of the net $\left(\pi_{\alpha}\right)$.

Conversely, assume the net of positive orthomorphisms $\pi_{\alpha} \stackrel{\circ}{\rightarrow} \pi$ in $\operatorname{Orth}(E)$. Then we have $\pi_{\alpha}(f) \stackrel{\mathrm{o}}{\rightarrow} \pi(f)$ for all $f \in E$; see Theorem VIII.2.3 [7]. For fixed $0 \leq \mu \in \operatorname{Orth}(E)$, there is a reel number $\lambda_{\mu}$ such that $\mu(f)=\lambda_{\mu} f$ for all $f \in E$. Since $\left|\pi_{\alpha}(f)-\pi(f)\right|=$ $\left|\lambda_{\pi_{\alpha}} f-\lambda_{\pi} f\right| \stackrel{\mathrm{o}}{\rightarrow} 0$, we have

$$
\left|\left(\pi_{\alpha}\right) f-(\pi) f\right| \mu=\left|\mu \lambda_{\pi_{\alpha}} f-\mu \lambda_{\pi} f\right|=\left|\lambda_{\mu} \lambda_{\pi_{\alpha}} f-\lambda_{\mu} \lambda_{\pi} f\right|=\left|\lambda_{\mu}\right|\left|\lambda_{\pi_{\alpha}} f-\lambda_{\pi} f\right| \stackrel{\mathrm{o}}{\rightarrow} 0
$$

for all $f \in E$. Since $\mu$ is arbitrary, we get $\pi_{\alpha} \stackrel{\mathrm{mo}}{\longrightarrow} \pi$.

\section{Main results}

We begin the section with the next list of properties of the mo-convergence which follows directly from Lemma 1.3, and the inequalities $|x-y| \leq\left|x-x_{\alpha}\right|+\left|x_{\alpha}-y\right|$ and ||$x_{\alpha}|-| x|| \leq\left|x_{\alpha}-x\right|$.

Lemma 2.1. Let $x_{\alpha} \stackrel{\mathrm{mo}}{\longrightarrow} x$ and $y_{\alpha} \stackrel{\mathrm{mo}}{\longrightarrow} y$ in an $f$-algebra $E$. Then the following holds:

(i) $x_{\alpha} \stackrel{\mathrm{mo}}{\longrightarrow} x$ if and only if $\left(x_{\alpha}-x\right) \stackrel{\mathrm{mo}}{\longrightarrow} 0$;

(ii) if $x_{\alpha} \stackrel{\mathrm{mo}}{\longrightarrow} x$ then $y_{\beta} \stackrel{\mathrm{mo}}{\longrightarrow} x$ for each subnet $\left(y_{\beta}\right)$ of $\left(x_{\alpha}\right)$;

(iii) suppose $x_{\alpha} \stackrel{\mathrm{mo}}{\longrightarrow} x$ and $y_{\beta} \stackrel{\mathrm{mo}}{\longrightarrow} y$ then $a x_{\alpha}+b y_{\beta} \stackrel{\mathrm{mo}}{\longrightarrow} a x+$ by for any $a, b \in \mathbb{R}$;

(iv) if $x_{\alpha} \stackrel{\mathrm{mo}}{\longrightarrow} x$ and $x_{\alpha} \stackrel{\mathrm{mo}}{\longrightarrow} y$ then $x=y$;

(v) if $x_{\alpha} \stackrel{\mathrm{mo}}{\longrightarrow} x$ then $\left|x_{\alpha}\right| \stackrel{\mathrm{mo}}{\longrightarrow}|x|$.

Recall that an order complete vector lattice $E^{\delta}$ is said to be an order completion of the vector lattice $E$ whenever $E$ is Riesz isomorphic to a majorizing order dense vector lattice subspace of $E^{\delta}$. Every Archimedean Riesz space has a unique order completion; see Theorem 2.24 [1].

Proposition 2.2. Let $\left(x_{\alpha}\right)$ be a net in an $f$-algebra $E$. Then $x_{\alpha} \stackrel{\mathrm{mo}}{\longrightarrow} 0$ in $E$ if and only if $x_{\alpha} \stackrel{\mathrm{mo}}{\longrightarrow} 0$ in the order completion $E^{\delta}$ of $E$.

Proof. Assume $x_{\alpha} \stackrel{\mathrm{mo}}{\longrightarrow} 0$ in $E$. Then $\left|x_{\alpha}\right| u \stackrel{\circ}{\rightarrow} 0$ in $E$ for all $u \in E_{+}$, and so $\left|x_{\alpha}\right| u \stackrel{\circ}{\rightarrow} 0$ in $E^{\delta}$ for all $u \in E_{+}$; see Corollary 2.9 [6]. Now, let us fix $v \in E_{+}^{\delta}$. Then there exists $x_{v} \in E_{+}$such that $v \leq x_{v}$ because $E$ majorizes $E^{\delta}$. Then we have $\left|x_{\alpha}\right| v \leq\left|x_{\alpha}\right| x_{v}$. From $\left|x_{\alpha}\right| x_{v} \stackrel{\circ}{\rightarrow} 0$ in $E^{\delta}$ it follows that $\left|x_{\alpha}\right| v \stackrel{\circ}{\rightarrow} 0$ in $E^{\delta}$, that is, $x_{\alpha} \stackrel{\text { mo }}{\longrightarrow} 0$ in the order completion $E^{\delta}$ because $v \in E_{+}^{\delta}$ is arbitrary.

Conversely, assume $x_{\alpha} \stackrel{\text { mo }}{\longrightarrow} 0$ in $E^{\delta}$. Then, for all $u \in E_{+}^{\delta}$, we have $\left|x_{\alpha}\right| u \stackrel{\circ}{\rightarrow} 0$ in $E^{\delta}$. In particular, for all $x \in E_{+},\left|x_{\alpha}\right| x \stackrel{\circ}{\rightarrow} 0$ in $E^{\delta}$. By Corollary 2.9 [6], we get $\left|x_{\alpha}\right| x \stackrel{\circ}{\rightarrow} 0$ in $E$ for all $x \in E_{+}$. Hence $x_{\alpha} \stackrel{\text { mo }}{\longrightarrow}$ in $E$.

The multiplication in $f$-algebra is mo-continuous in the following sense.

Theorem 2.3. Let $E$ be an $f$-algebra, and $\left(x_{\alpha}\right)_{\alpha \in A}$ and $\left(y_{\beta}\right)_{\beta \in B}$ be two nets in $E$. If $x_{\alpha} \stackrel{\mathrm{mo}}{\longrightarrow} x$ and $y_{\beta} \stackrel{\mathrm{mo}}{\longrightarrow} y$ for some $x, y \in E$ and each positive element of $E$ can be written as a multiplication of two positive elements then $x_{\alpha} y_{\beta} \stackrel{\mathrm{mo}}{\longrightarrow} x y$. 
Proof. Assume $x_{\alpha} \stackrel{\mathrm{mo}}{\longrightarrow} x$ and $y_{\beta} \stackrel{\mathrm{mo}}{\longrightarrow} y$. Then $\left|x_{\alpha}-x\right| u \stackrel{\mathrm{o}}{\rightarrow} 0$ and $\left|y_{\beta}-y\right| u \stackrel{\circ}{\rightarrow} 0$ for every $u \in E_{+}$. Let us fix $u \in E_{+}$. So, there exist another two nets $\left(z_{\gamma}\right)_{\gamma \in \Gamma} \downarrow 0$ and $\left(z_{\xi}\right)_{\xi \in \Xi \downarrow 0}$ in $E$ such that, for all $(\gamma, \xi) \in \Gamma \times \Xi$ there are $\alpha_{\gamma} \in A$ and $\beta_{\xi} \in B$ with $\left|x_{\alpha}-x\right| u \leq z_{\gamma}$ and $\left|y_{\beta}-y\right| u \leq z_{\xi}$ for all $\alpha \geq \alpha_{\gamma}$ and $\beta \geq \beta_{\xi}$.

Next, we show the mo-convergence of $\left(x_{\alpha} y_{\beta}\right)$ to $x y$. By considering the equality $|x y|=$ $|x||y|$ and Lemma 1.3, we have

$$
\begin{aligned}
\left|x_{\alpha} y_{\beta}-x y\right| u & =\left|x_{\alpha} y_{\beta}-x_{\alpha} y+x_{\alpha} y-x y\right| u \\
& \leq\left|x_{\alpha}\right|\left|y_{\beta}-y\right| u+\left|x_{\alpha}-x\right||y| u \\
& \leq\left|x_{\alpha}-x\right|\left|y_{\beta}-y\right| u+|x|\left|y_{\beta}-y\right| u+\left|x_{\alpha}-x\right||y| u .
\end{aligned}
$$

The second and the third terms in the last inequality both order converge to zero as $\beta \rightarrow \infty$ and $\alpha \rightarrow \infty$ respectively because of $|x| u,|y| u \in E_{+}, x_{\alpha} \stackrel{\mathrm{mo}}{\longrightarrow} x$ and $y_{\beta} \stackrel{\mathrm{mo}}{\longrightarrow} y$.

Now, let us show the convergence of the first term of last inequality. There are two positive elements $u_{1}, u_{2} \in E_{+}$such that $u=u_{1} u_{2}$ because the positive element of $E$ can be written as a multiplication of two positive elements. So, we get $\left|x_{\alpha}-x\right|\left|y_{\beta}-y\right| u=$

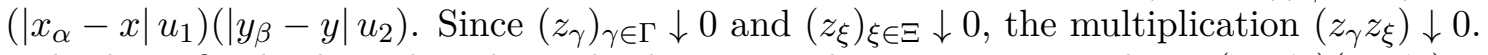
Indeed, we firstly show that the multiplication is decreasing. For indexes $\left(\gamma_{1}, \xi_{1}\right)\left(\gamma_{2}, \xi_{2}\right) \in$ $\Gamma \times \Xi$, we have $z_{\gamma_{2}} \leq z_{\gamma_{1}}$ and $z_{\xi_{2}} \leq z_{\xi_{1}}$ because both of them are decreasing. Since the nets are positive, it follows from $z_{\xi_{2}} \leq z_{\xi_{1}}$ that $z_{\gamma_{2}} z_{\xi_{2}} \leq z_{\gamma_{2}} z_{\xi_{1}} \leq z_{\gamma_{1}} z_{\xi_{1}}$. As

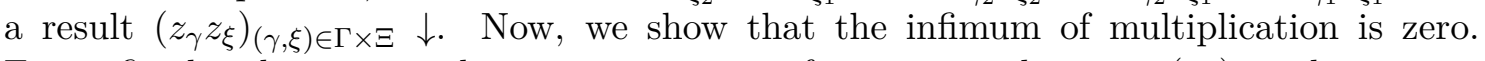
For a fixed index $\gamma_{0}$, we have $z_{\gamma} z_{\xi} \leq z_{\gamma_{0}} z_{\xi}$ for $\gamma \geq \gamma_{0}$ because $\left(z_{\gamma}\right)$ is decreasing. Thus, we get $\inf \left(z_{\gamma} z_{\xi}\right)=0$ because of $\inf \left(z_{\gamma_{0}} z_{\xi}\right)=z_{\gamma_{0}} \inf \left(z_{\xi}\right)=0$. Therefore, we see $\left(\left|x_{\alpha}-x\right| u_{1}\right)\left(\left|y_{\beta}-y\right| u_{2}\right) \stackrel{\mathrm{o}}{\rightarrow} 0$. Hence, we get $x_{\alpha} y_{\beta} \stackrel{\mathrm{mo}}{\longrightarrow} x y$.

The lattice operations in an $f$-algebra are mo-continuous in the following sense.

Proposition 2.4. Let $\left(x_{\alpha}\right)_{\alpha \in A}$ and $\left(y_{\beta}\right)_{\beta \in B}$ be two nets in an $f$-algebra E. If $x_{\alpha} \stackrel{\text { mo }}{\longrightarrow} x$ and $y_{\beta} \stackrel{\mathrm{mo}}{\longrightarrow} y$ then $\left(x_{\alpha} \vee y_{\beta}\right)_{(\alpha, \beta) \in A \times B} \stackrel{\mathrm{mo}}{\longrightarrow} x \vee y$. In particular, $x_{\alpha} \stackrel{\mathrm{mo}}{\longrightarrow} x$ implies $x_{\alpha}^{+} \stackrel{\mathrm{mo}}{\longrightarrow} x^{+}$.

Proof. Assume $x_{\alpha} \stackrel{\mathrm{mo}}{\longrightarrow} x$ and $y_{\beta} \stackrel{\mathrm{mo}}{\longrightarrow} y$. Then there exist two nets $\left(z_{\gamma}\right)_{\gamma \in \Gamma}$ and $\left(w_{\lambda}\right)_{\lambda \in \Lambda}$ in $E$ satisfying $z_{\gamma} \downarrow 0$ and $w_{\lambda} \downarrow 0$, and for all $(\gamma, \lambda) \in \Gamma \times \Lambda$ there are $\alpha_{\gamma} \in A$ and $\beta_{\lambda} \in B$ such that $\left|x_{\alpha}-x\right| u \leq z_{\gamma}$ and $\left|y_{\beta}-y\right| u \leq w_{\lambda}$ for all $\alpha \geq \alpha_{\gamma}$ and $\beta \geq \beta_{\lambda}$ and for every $u \in E_{+}$. It follows from the inequality $|a \vee b-a \vee c| \leq|b-c|$ in vector lattices that

$$
\begin{aligned}
\left|x_{\alpha} \vee y_{\beta}-x \vee y\right| u & \leq\left|x_{\alpha} \vee y_{\beta}-x_{\alpha} \vee y\right| u+\left|x_{\alpha} \vee y-x \vee y\right| u \\
& \leq\left|y_{\beta}-y\right| u+\left|x_{\alpha}-x\right| u \leq w_{\lambda}+z_{\gamma}
\end{aligned}
$$

for all $\alpha \geq \alpha_{\gamma}$ and $\beta \geq \beta_{\lambda}$ and for every $u \in E_{+}$. Since $\left(w_{\lambda}+z_{\gamma}\right) \downarrow 0,\left|x_{\alpha} \vee y_{\beta}-x \vee y\right| u$ order converges to 0 for all $u \in E_{+}$. That is, $\left(x_{\alpha} \vee y_{\beta}\right)_{(\alpha, \beta) \in A \times B} \stackrel{\text { mo }}{\longrightarrow} x \vee y$.

Lemma 2.5. Let $\left(x_{\alpha}\right)$ be a net in an $f$-algebra $E$. Then

(i) $0 \leq x_{\alpha} \stackrel{\mathrm{mo}}{\longrightarrow} x$ implies $x \in E_{+}$.

(ii) if $\left(x_{\alpha}\right)$ is monotone and $x_{\alpha} \stackrel{\mathrm{mo}}{\longrightarrow} x$ then implies $x_{\alpha} \stackrel{\mathrm{o}}{\rightarrow} x$.

Proof. (i) Assume $0 \leq x_{\alpha} \stackrel{\text { mo }}{\longrightarrow} x$. Then we have $x_{\alpha}=x_{\alpha}^{+} \stackrel{\text { mo }}{\longrightarrow} x^{+}=x$ by Proposition 2.4. Hence, we get $x \in E_{+}$.

(ii) We show that $x_{\alpha} \uparrow$ and $x_{\alpha} \stackrel{\text { mo }}{\longrightarrow} x$ implies $x_{\alpha} \uparrow x$. Fix an index $\alpha$. Then we have $x_{\beta}-x_{\alpha} \in X_{+}$for $\beta \geq \alpha$. By $(i), x_{\beta}-x_{\alpha} \stackrel{\text { mo }}{\longrightarrow} x-x_{\alpha} \in X_{+}$. Therefore, $x \geq x_{\alpha}$ for any $\alpha$. Since $\alpha$ is arbitrary, then $x$ is an upper bound of $\left(x_{\alpha}\right)$. Assume $y$ is another upper bound of $\left(x_{\alpha}\right)$, i.e., $y \geq x_{\alpha}$ for all $\alpha$. So, $y-x_{\alpha} \stackrel{\text { mo }}{\longrightarrow} y-x \in X_{+}$, or $y \geq x$, and so $x_{\alpha} \uparrow x$.

The following simple observation is useful in its own right.

Proposition 2.6. Decreasing disjoint sequence in an $f$-algebra mo-converges to zero. 
Proof. Suppose $\left(x_{n}\right)$ is a disjoint decreasing sequence in an $f$-algebra $E$. So, $\left|x_{n}\right| u$ is also a disjoint sequence in $E$ for all $u \in E_{+}$; see Theorem 142.1(iii) [8]. Fix $u \in E_{+}$, by Corollary 3.6 [6], we have $\left|x_{n}\right| u \stackrel{\text { uo }}{\longrightarrow} 0$ in $E$. So, $\left|x_{n}\right| u \wedge w \stackrel{\mathrm{o}}{\rightarrow} 0$ in $E$ for all $w \in E_{+}$. Thus, in particular, for fixed $n_{0}$, taking $w$ as $\left|x_{n_{0}}\right| u$, Then, for all $n \geq n_{0}$, we get

$$
\left|x_{n}\right| u=\left|x_{n}\right| u \wedge\left|x_{n_{0}}\right| u=\left|x_{n}\right| u \wedge w \stackrel{\circ}{\rightarrow} 0 .
$$

because of $\left|x_{n}\right| u \leq\left|x_{n_{0}}\right| u$. Therefore, $x_{n} \stackrel{\mathrm{mo}}{\longrightarrow} 0$ in $E$.

For the next two facts, observe the following fact. Let $E$ be a vector lattice, $I$ be an order ideal of $E$ and $\left(x_{\alpha}\right)$ be a net in $I$. If $x_{\alpha} \stackrel{\circ}{\rightarrow} x$ in $I$ then $x_{\alpha} \stackrel{\circ}{\rightarrow} x$ in $E$. Conversely, if $\left(x_{\alpha}\right)$ is order bounded in $I$ and $x_{\alpha} \stackrel{\circ}{\rightarrow} x$ in $E$ then $x_{\alpha} \stackrel{\circ}{\rightarrow} x$ in $I$.

Proposition 2.7. Let $E$ be an $f$-algebra, $B$ be a projection band of $E$ and $P_{B}$ be the corresponding band projection. If $x_{\alpha} \stackrel{\mathrm{mo}}{\longrightarrow} x$ in $E$ then $P_{B}\left(x_{\alpha}\right) \stackrel{\mathrm{mo}}{\longrightarrow} P_{B}(x)$ in both $E$ and $B$.

Proof. It is known that $P_{B}$ is a lattice homomorphism and $0 \leq P_{B} \leq I$. It follows from $\left|P_{B}\left(x_{\alpha}\right)-P_{B}(x)\right|=P_{B}\left|x_{\alpha}-x\right| \leq\left|x_{\alpha}-x\right|$ that $\left|P_{B}\left(x_{\alpha}\right)-P_{B}(x)\right| u \leq\left|x_{\alpha}-x\right| u$ for all $u \in E_{+}$. Then it follows easily that $P_{B}\left(x_{\alpha}\right) \stackrel{\text { mo }}{\longrightarrow} P_{B}(x)$ in both $X$ and $B$.

Theorem 2.8. Let $E$ be an $f$-algebra and $I$ be an order ideal and sub-f-algebra of $E$. For an order bounded net $\left(x_{\alpha}\right)$ in $I, x_{\alpha} \stackrel{\mathrm{mo}}{\longrightarrow} 0$ in $I$ if and only if $x_{\alpha} \stackrel{\mathrm{mo}}{\longrightarrow} 0$ in $E$.

Proof. Suppose $x_{\alpha} \stackrel{\text { mo }}{\longrightarrow} 0$ in $E$. Then for any $u \in I_{+}$, we have $\left|x_{\alpha}\right| u \stackrel{\circ}{\rightarrow} 0$ in $E$. So, the preceding remark implies $\left|x_{\alpha}\right| u \stackrel{\circ}{\rightarrow} 0$ in $I$ because $\left|x_{\alpha}\right| u$ is order bounded in $I$. Therefore, we get $x_{\alpha} \stackrel{\mathrm{mo}}{\longrightarrow} 0$ in $I$.

Conversely, assume that $\left(x_{\alpha}\right)$ mo-converges to zero in $I$. For any $u \in I_{+}$, we have $\left|x_{\alpha}\right| u \stackrel{\mathrm{o}}{\rightarrow} 0$ in $I$, and so in $E$. Then, by applying Theorem 142.1(iv) [8], we have $x_{\alpha} w=0$ for all $w \in I^{d}=\{x \in E: x \perp y$ for all $y \in I\}$ and for each $\alpha$ because $\left(x_{\alpha}\right)$ in $I$. For any $u \in I_{+}$and each $0 \leq w \in I^{d}$, it follows that

$$
\left|x_{\alpha}\right|(u+w)=\left|x_{\alpha}\right| u+\left|x_{\alpha}\right| w=\left|x_{\alpha}\right| u \stackrel{\circ}{\rightarrow} 0
$$

in $E$. So that, for each $z \in\left(I \oplus I^{d}\right)_{+}$, we get $\left|x_{\alpha}\right| z \stackrel{\circ}{\rightarrow} 0$ in $E$. It is known that $I \oplus I^{d}$ is order dense in $E$; see Theorem 1.36 [1]. Fix $v \in E_{+}$. Then there exists some $u \in\left(I \oplus I^{d}\right)$ such that $v \leq u$. Thus, we have $\left|x_{\alpha}\right| v \leq\left|x_{\alpha}\right| u \stackrel{\circ}{\rightarrow} 0$ in $E$. Therefore, $\left|x_{\alpha}\right| v \stackrel{\circ}{\rightarrow} 0$, and so $x_{\alpha} \stackrel{\mathrm{mo}}{\longrightarrow} 0$ in $E$.

The following proposition extends Theorem 3.8 [2] to the general setting.

Theorem 2.9. Let $E$ be an $f$-algebra with a unit e and $\left(x_{n}\right) \downarrow$ be a sequence in $E$. Then $x_{n} \stackrel{\mathrm{mo}}{\longrightarrow} 0$ if and only if $\left|x_{n}\right|(u \wedge e) \stackrel{\mathrm{o}}{\rightarrow} 0$ for all $u \in E_{+}$.

Proof. For the forward implication, assume $x_{n} \stackrel{\text { mo }}{\longrightarrow} 0$. Hence, $|x| u \stackrel{\circ}{\rightarrow} 0$ for all $u \in E_{+}$, and so $\left|x_{n}\right|(u \wedge e) \leq\left|x_{n}\right| u \stackrel{\circ}{\rightarrow} 0$ because of $e \in E_{+}$. Therefore, $\left|x_{n}\right|(u \wedge e) \stackrel{\circ}{\rightarrow} 0$.

For the reverse implication, fix $u \in E_{+}$. By applying Theorem 2.57 [1] and Theorem 142.1(i) [8], note that

$$
\left|x_{n}\right| u \leq\left|x_{n}\right|(u-u \wedge n e)+\left|x_{n}\right|(u \wedge n e) \leq \frac{1}{n} u^{2}\left|x_{n}\right|+n\left|x_{n}\right|(u \wedge e)
$$

Since $\left(x_{n}\right) \downarrow$ and $E$ is Archimedean, we have $\frac{1}{n} u^{2}\left|x_{n}\right| \downarrow 0$. Furthermore, it follows from $\left|x_{n}\right|(u \wedge e) \stackrel{\mathrm{O}}{\rightarrow} 0$ for each $u \in E_{+}$that there exists another sequence $\left(y_{m}\right)_{m \in B}$ satisfying $y_{m} \downarrow 0$, and for any $m \in B$, there exists $n_{m}$ such that $\left|x_{n}\right|(u \wedge e) \leq \frac{1}{n} y_{m}$, or $n\left|x_{n}\right|(u \wedge e) \leq$ $y_{m}$ for all $n \geq n_{m}$. Hence, we get $n\left|x_{n}\right|(u \wedge e) \stackrel{\circ}{\rightarrow} 0$. Therefore, we have $\left|x_{n}\right| u \stackrel{\circ}{\rightarrow} 0$, and so $x_{n} \stackrel{\mathrm{mo}}{\longrightarrow} 0$.

The mo-convergence passes obviously to any sub- $f$-algebra $Y$ of $E$, i.e., for any net $\left(y_{\alpha}\right)$ in $Y, y_{\alpha} \stackrel{\mathrm{mo}}{\longrightarrow} 0$ in $E$ implies $y_{\alpha} \stackrel{\mathrm{mo}}{\longrightarrow} 0$ in $Y$. For the converse, we give the following theorem. 
Theorem 2.10. Let $Y$ be a sub-f-algebra of an $f$-algebra $E$ and $\left(y_{\alpha}\right)$ be a net in $Y$. If $y_{\alpha} \stackrel{\mathrm{mo}}{\longrightarrow} 0$ in $Y$ then it mo-converges to zero in $E$ for each of the following cases;

(i) $Y$ is majorizing in $E$;

(ii) $Y$ is a projection band in $E$;

(iii) if, for each $u \in E$, there are element $x, y \in Y$ such that $|u-y| \leq|x|$.

Proof. Assume $\left(y_{\alpha}\right)$ is a net in $Y$ and $y_{\alpha} \stackrel{\text { mo }}{\longrightarrow} 0$ in $Y$. Let us fix $u \in E_{+}$.

(i) Since $Y$ is majorizing in $E$, there exists $v \in Y_{+}$such that $u \leq v$. It follows from

$$
0 \leq\left|y_{\alpha}\right| u \leq\left|y_{\alpha}\right| v \stackrel{\circ}{\rightarrow} 0,
$$

that $\left|y_{\alpha}\right| u \stackrel{\circ}{\rightarrow} 0$ in $E$. That is, $y_{\alpha} \stackrel{\mathrm{mo}}{\longrightarrow} 0$ in $E$.

(ii) Since $Y$ is a projection band in $E$, we have $Y=Y^{\perp \perp}$ and $E=Y \oplus Y^{\perp}$. Hence $u=u_{1}+u_{2}$ with $u_{1} \in Y_{+}$and $u_{2} \in Y_{+}^{\perp}$. Thus, we have $y_{\alpha} \wedge u_{2}=0$ because $\left(y_{\alpha}\right)$ in $Y$ and $u_{2} \in Y^{\perp}$. Hence, by applying Theorem 142.1(iii) [8], we see $y_{\alpha} u=0$ for all index $\alpha$. It follows from

$$
\left|y_{\alpha}\right| u=\left|y_{\alpha}\right|\left(u_{1}+u_{2}\right)=\left|y_{\alpha}\right| u_{1} \stackrel{\circ}{\rightarrow} 0
$$

that $\left|y_{\alpha}\right| u \stackrel{\mathrm{o}}{\rightarrow} 0$ in $E$. Therefore, $y_{\alpha} \stackrel{\mathrm{mo}}{\longrightarrow} 0$ in $E$.

(iii) For the given $u \in E_{+}$, there exists elements $x, y \in Y$ with $|u-y| \leq|x|$. Then

$$
\left|y_{\alpha}\right| u \leq\left|y_{\alpha}\right||u-y|+\left|y_{\alpha}\right||y| \leq\left|y_{\alpha}\right||x|+\left|y_{\alpha}\right||y| \text {. }
$$

By mo-convergence of $\left(y_{\alpha}\right)$ in $Y$, we have $\left|y_{\alpha}\right||x| \stackrel{\circ}{\rightarrow} 0$ and $\left|y_{\alpha}\right||y| \stackrel{\circ}{\rightarrow} 0$, and so $\left|y_{\alpha}\right| u \stackrel{\circ}{\rightarrow} 0$. That means $y_{\alpha} \stackrel{\mathrm{mo}}{\longrightarrow} 0$ in $E$ because $u$ is arbitrary in $E_{+}$.

We continue with some basic notions in $f$-algebra, which are motivated by their analogies from vector lattice theory.

Definition 2.11. Let $\left(x_{\alpha}\right)_{\alpha \in A}$ be a net in $f$-algebra $E$. Then

(i) $\left(x_{\alpha}\right)$ is said to be mo-Cauchy if the net $\left(x_{\alpha}-x_{\alpha^{\prime}}\right)_{\left(\alpha, \alpha^{\prime}\right) \in A \times A}$ mo-converges to 0 ,

(ii) $E$ is called mo-complete if every mo-Cauchy net in $E$ is mo-convergent,

(iii) $E$ is called mo-continuous if $x_{\alpha} \stackrel{\mathrm{o}}{\rightarrow} 0$ implies $x_{\alpha} \stackrel{\mathrm{mo}}{\longrightarrow} 0$,

(iv) $E$ is called a mo-KB-space if every order bounded increasing net in $E_{+}$is $m o-$ convergent.

Remark 2.12. An $f$-algebra $E$ is mo-continuous if and only if $x_{\alpha} \downarrow 0$ in $E$ implies $x_{\alpha} \stackrel{\text { mo }}{\longrightarrow} 0$. Indeed, the implication is obvious. For the converse, consider a net $x_{\alpha} \stackrel{\mathrm{o}}{\rightarrow} 0$. Then there exists a net $z_{\beta} \downarrow 0$ in $X$ such that, for any $\beta$ there exists $\alpha_{\beta}$ so that $\left|x_{\alpha}\right| \leq z_{\beta}$ for all $\alpha \geq \alpha_{\beta}$. Hence, by mo-continuity of $E$, we have $z_{\beta} \stackrel{\text { mo }}{\longrightarrow} 0$, and so $x_{\alpha} \stackrel{\text { mo }}{\longrightarrow} 0$.

Proposition 2.13. Let $\left(x_{\alpha}\right)$ be a net in an f-algebra $E$. If $x_{\alpha} \stackrel{\mathrm{mo}}{\longrightarrow} x$ and $\left(x_{\alpha}\right)$ is an $o$-Cauchy net then $x_{\alpha} \stackrel{\mathrm{o}}{\rightarrow} x$. Moreover, if $x_{\alpha} \stackrel{\mathrm{mo}}{\longrightarrow} x$ and $\left(x_{\alpha}\right)$ is uo-Cauchy then $x_{\alpha} \stackrel{\mathrm{uo}}{\longrightarrow} x$.

Proof. Assume $x_{\alpha} \stackrel{\mathrm{mo}}{\longrightarrow} x$ and $\left(x_{\alpha}\right)$ is an order Cauchy net in $E$. Then $x_{\alpha}-x_{\beta} \stackrel{\circ}{\rightarrow} 0$ as $\alpha, \beta \rightarrow \infty$. Thus, there exists another net $z_{\gamma} \downarrow 0$ in $E$ such that, for every $\gamma$, there exists $\alpha_{\gamma}$ satisfying

$$
\left|x_{\alpha}-x_{\beta}\right| \leq z_{\gamma}
$$

for all $\alpha, \beta \geq \alpha_{\gamma}$. By taking $f$-limit over $\beta$ the above inequality and applying Proposition 2.4, i.e., $\left|x_{\alpha}-x_{\beta}\right| \stackrel{\mathrm{mo}}{\longrightarrow}\left|x_{\alpha}-x\right|$, we get $\left|x_{\alpha}-x\right| \leq z_{\gamma}$ for all $\alpha \geq \alpha_{\gamma}$. That means $x_{\alpha} \stackrel{\mathrm{o}}{\rightarrow} x$. The similar argument can be applied for the uo-convergence case, and so the proof is omitted.

In the case of $m o$-complete in $f$-algebras, we have conditions for mo-continuity.

Theorem 2.14. For an mo-complete $f$-algebra $E$, the following statements are equivalent:

(i) E is mo-continuous; 
(ii) if $0 \leq x_{\alpha} \uparrow \leq x$ holds in $E$ then $x_{\alpha}$ is a mo-Cauchy net;

(iii) $x_{\alpha} \downarrow 0$ implies $x_{\alpha} \stackrel{\mathrm{mo}}{\longrightarrow} 0$ in $E$.

Proof. $(i) \Rightarrow($ ii $)$ Consider the increasing and bounded net $0 \leq x_{\alpha} \uparrow \leq x$ in $E$. Then there exists a net $\left(y_{\beta}\right)$ in $E$ such that $\left(y_{\beta}-x_{\alpha}\right)_{\alpha, \beta} \downarrow 0$; see Lemma 12.8 [1]. Thus, by applying Remark 2.12, we have $\left(y_{\beta}-x_{\alpha}\right)_{\alpha, \beta} \stackrel{\text { mo }}{\longrightarrow} 0$, and so the net $\left(x_{\alpha}\right)$ is mo-Cauchy because of $\left|x_{\alpha}-x_{\alpha^{\prime}}\right|_{\alpha, \alpha^{\prime} \in A} \leq\left|x_{\alpha}-y_{\beta}\right|+\left|y_{\beta}-x_{\alpha^{\prime}}\right|$.

(ii) $\Rightarrow$ (iii) Suppose that $x_{\alpha} \downarrow 0$ in $E$, and fix arbitrary $\alpha_{0}$. Then we have $x_{\alpha} \leq x_{\alpha_{0}}$ for all $\alpha \geq \alpha_{0}$. Thus we can get $0 \leq\left(x_{\alpha_{0}}-x_{\alpha}\right)_{\alpha \geq \alpha_{0}} \uparrow \leq x_{\alpha_{0}}$. So, it follows from (ii) that the net $\left(x_{\alpha_{0}}-x_{\alpha}\right)_{\alpha \geq \alpha_{0}}$ is mo-Cauchy, i.e., $\left(x_{\alpha^{\prime}}-x_{\alpha}\right) \stackrel{\text { mo }}{\longrightarrow} 0$ as $\alpha_{0} \leq \alpha, \alpha^{\prime} \rightarrow \infty$. Then there exists $x \in E$ satisfying $x_{\alpha} \stackrel{\text { mo }}{\longrightarrow} x$ as $\alpha_{0} \leq \alpha \rightarrow \infty$ because $E$ is mo-complete. Since $x_{\alpha} \downarrow$ and $x_{\alpha} \stackrel{\mathrm{mo}}{\longrightarrow} 0$, it follows from Lemma 2.5 that $x_{\alpha} \downarrow 0$, and so we have $x=0$. Therefore, we get $x_{\alpha} \stackrel{\text { mo }}{\longrightarrow} 0$.

$($ iii $) \Rightarrow($ i) It is just the implication of Remark 2.12.

Corollary 2.15. Let $E$ be an mo-continuous and mo-complete $f$-algebra. Then $E$ is order complete.

Proof. Suppose $0 \leq x_{\alpha} \uparrow \leq u$ in $E$. We show the existence of supremum of $\left(x_{\alpha}\right)$. By considering Theorem $2.14(\mathrm{ii})$, we see that $\left(x_{\alpha}\right)$ is an mo-Cauchy net. Hence, there is $x \in E$ such that $x_{\alpha} \stackrel{\text { mo }}{\longrightarrow} x$ because $E$ is mo-complete. It follows from Lemma 2.5 that $x_{\alpha} \uparrow x$ because of $x_{\alpha} \uparrow$ and $x_{\alpha} \stackrel{\mathrm{mo}}{\longrightarrow} x$. Therefore, $E$ is order complete.

Proposition 2.16. Every mo-KB-space is mo-continuous.

Proof. Assume $x_{\alpha} \downarrow 0$ in E. From Theorem 2.14, it is enough to show $x_{\alpha} \stackrel{\text { mo }}{\longrightarrow} 0$. Let us fix an index $\alpha_{0}$, and define another net $y_{\alpha}:=x_{\alpha_{0}}-x_{\alpha}$ for $\alpha \geq \alpha_{0}$. Then it is clear that $0 \leq y_{\alpha} \uparrow \leq x_{\alpha_{0}}$, i.e., $\left(y_{\alpha}\right)$ is increasing and order bounded net in $E$. Since $E$ is a mo-KB-space, there exists $y \in E$ such that $y_{\alpha} \stackrel{\text { mo }}{\longrightarrow} y$. Thus, by Lemma 2.5, we have $y_{\alpha} \stackrel{\circ}{\rightarrow} y$. Hence, $y=\sup _{\alpha \geq \alpha_{0}} y_{\alpha}=\sup _{\alpha \geq \alpha_{0}}\left(x_{\alpha_{0}}-x_{\alpha}\right)=x_{\alpha_{0}}$ because of $x_{\alpha} \downarrow 0$. Therefore, we get $y_{\alpha}=x_{\alpha_{0}}-x_{\alpha} \stackrel{\mathrm{mo}}{\longrightarrow} x_{\alpha_{0}}$ or $x_{\alpha} \stackrel{\mathrm{mo}}{\longrightarrow} 0$ because of $y_{\alpha} \stackrel{\mathrm{mo}}{\longrightarrow} y$.

Proposition 2.17. Every mo-KB-space is order complete.

Proof. Suppose $0 \leq x_{\alpha} \uparrow \leq z$ is an order bounded and increasing net in an mo-KB-space $E$ for some $z \in E_{+}$. Then $x_{\alpha} \stackrel{\text { mo }}{\longrightarrow} x$ for some $x \in E$ because $E$ is $m o-\mathrm{KB}$-space. By Lemma 2.5 , we have $x_{\alpha} \uparrow x$ because of $x_{\alpha} \uparrow$ and $x_{\alpha} \stackrel{\text { mo }}{\longrightarrow} x$. So, $E$ is order complete.

Proposition 2.18. Let $Y$ be an sub-f-algebra and order closed sublattice of an mo-KBspace $E$. Then $Y$ is also a mo-KB-space.

Proof. Let $\left(y_{\alpha}\right)$ be a net in $Y$ such that $0 \leq y_{\alpha} \uparrow \leq y$ for some $y \in Y_{+}$. Since $E$ is a $m o-K B-s p a c e$, there exists $x \in E_{+}$such that $y_{\alpha} \stackrel{\text { mo }}{\longrightarrow} x$. By Lemma 2.5, we have $y_{\alpha} \uparrow x$, and so $x \in Y$ because $Y$ is order closed. Thus $Y$ is a $m o-\mathrm{KB}$-space.

\section{References}

[1] C.D. Aliprantis and O. Burkinshaw, Positive Operators, Springer, Dordrecht, 2006.

[2] A. Aydın, Unbounded $p_{\tau}$-convergence in vector lattice normed by locally solid lattices, Academic Studies in Mathematic and Natural Sciences-2019/2, 118-134, IVPE, Cetinje-Montenegro, 2019.

[3] A. Aydın, S.G. Gorokhova and H. Gül, Nonstandard hulls of lattice-normed ordered vector spaces, Turkish J. Math. 42 (1), 155-163, 2018.

[4] A. Aydın, E. Emel'yanov, N. Erkurşun-Özcan and M.A.A. Marabeh, Compact-like operators in lattice-normed spaces, Indag. Math. 2 (1), 633-656, 2018. 
[5] A. Aydin, E. Emel'yanov, N. Erkurşun-Özcan and M.A.A. Marabeh, Unbounded pconvergence in lattice-normed vector lattices, Sib. Adv. Math. 29 (3), 164-182, 2019.

[6] N. Gao, V.G. Troitsky and F. Xanthos, Uo-convergence and its applications to Cesáro means in Banach lattices, Isr. J. Math. 220 (2), 649-689, 2017.

[7] B.Z. Vulikh, Introduction to the Theory of Partially Ordered Spaces, WoltersNoordhoff Scientific, Groningen, 1967.

[8] A.C. Zaanen, Riesz Spaces II, North-Holland Publishing C., Amsterdam, 1983. 